\title{
Morus nigra $L .:$ revisão sistematizada das propriedades botânicas, fitoquímicas e farmacológicas
}

\author{
Morus nigra L.: systematized review of botanical, phytochemical and pharmacological properties
}

Morus nigra L.: revisión sistematizada de las propiedades botánicas, fitoquímicas y farmacológicas

Tatiane Nelí Ferreira Lima de OLIVEIRA ${ }^{1}$ Cássia Cardoso COSTA ${ }^{2}$

Danielle de Pontes ESTEVAM ${ }^{1}$

Iris Anunciação dos Anjos MEDEIROS

Ericlebson Cleyton da Silva LIMA ${ }^{3}$

Venâncio Mike SANTOS ${ }^{4}$

Abrahão Alves de OLIVEIRA FILHO 5

Heloísa Mara Batista Fernandes de OLIVEIRA ${ }^{6}$

${ }^{1}$ Graduanda, Bacharelado em Farmácia, Centro de Ciências da Saúde, Universidade Federal do Rio Grande do Norte-UFRN, 59012-570 Natal-RN, Brasil ${ }^{2}$ Residência Multiprofissional, Hospital Universitário Ana Bezerra, Universidade Federal do Rio Grande do Norte-UFRN, 59012-570 Natal-RN, Brasil ${ }^{3}$ Graduando(a), Bacharelado em Farmácia, Centro de Educação e Saúde, Universidade Federal de Campina Grande-UFCG, 58175-000 Cuité-PB, Brasil ${ }^{4}$ Graduando, Bacharelado em Biomedicina, Centro de Biociências, Universidade Federal do Rio Grande do Norte-UFRN, 59064-741 Natal-RN, Brasil ${ }^{5}$ Professor da Unidade Acadêmica de Ciências Biológicas-UACB, Centro de Saúde e Tecnologia Rural, Universidade Federal de Campina Grande- UFCG, 58700-970 Patos-PB, Brasil

${ }^{6}$ Farmacêutica - Bioquímica - Doutora em Farmacologia, Hospital Universitário Ana Bezerra - HUAB/EBSERH, 59200-000 Santa Cruz-RN, Brasil

\section{Resumo}

Morus nigra é uma espécie vegetal popularmente conhecida por Amora negra. Esta espécie amplamente cultivada na Ásia e nas regiões sul e sudeste do Brasil, possui importância econômica devido a sua ampla utilização na alimentação e confeitaria, sendo também utilizada na medicina popular como anti-inflamatório, diurético, antitussígeno, anti-hemorrágico, para o tratamento do climatério e de doenças hepáticas e renais. Diversos estudos têm sido conduzidos a respeito das atividades farmacológicas da Morus nigra. Neste trabalho de revisão narrativa da literatura foram explorados estudos nas bases de dados acerca da atividade hipolipemiante e de outras atividades farmacológicas dos compostos fenólicos, estilbenos, flavonoide, isoprenilados, cumarinas, cromonas e xantonas da Morus nigra, para verificar sua capacidade de diminuição dos níveis de colesterol total, triglicerídeos, LDL e ainda, aumento dos níveis de HDL, como também outras propriedades antioxidantes, anticarcinogênica, antimutagênica. Desse modo, conclui-se a necessidade da realização de mais estudos com Morus nigra e principalmente mais ensaios clínicos que avaliem a segurança e eficácia na utilização desta planta.

Descritores: Farmacologia; Fitoterapia; Hiperlipidemias; Morus.

\section{Abstract}

Morus nigra is a plant species popularly known as blackberry. This species, widely cultivated in Asia and in the south and southeast regions of Brazil, is economically important due to its wide use in food and confectionery. It is also used in folk medicine as anti-inflammatory, diuretic, antitussive, anti-hemorrhagic, for treatment climacteric and liver and kidney diseases. Several studies have been conducted regarding the pharmacological activities of Morus nigra. In this work of narrative review of the literature we explored studies in the databases on the lipid-lowering activity and other pharmacological activities of the phenolic, stilbenos, flavonoids, isoprenylates, coumarins, cromones and xanthones compounds of Morus nigra, to verify their capacity to decrease levels of total cholesterol, triglycerides, LDL and also, increased levels of HDL, as well as other antioxidant properties, anticarcinogenic, antimutagenic. Thus, it is concluded that further studies are needed with Morus nigra and, in particular, more clinical trials evaluating the safety and efficacy of this plant.

Descriptors: Pharmacology; Phytotherapy; Hyperlipidemias; Morus.

\section{Resumen}

Morus nigra es una especie vegetal popularmente conocida por Amora negra. Esta especie ampliamente cultivada en Asia y en las regiones sur y sudeste de Brasil, tiene una importancia económica debido a su amplia utilización en la alimentación y la confitería, siendo utilizada en la medicina popular como anti-inflamatorio, diurético, antitusígeno, anti-hemorrágico, para el tratamiento del climaterio y de las enfermedades hepáticas y renales. Se han realizado varios estudios sobre las actividades farmacológicas de Morus nigra. En este trabajo de revisión narrativa de la literatura se exploraron estudios en las bases de datos acerca de la actividad hipolipemiante y de otras actividades farmacológicas de los compuestos fenólicos, estilbenos, flavonoide, isoprenilados, cumarinas, cromonas y xantonas de Morus nigra, para verificar su capacidad de disminución de los niveles de colesterol total, triglicéridos, LDL y aún, aumento de los niveles de HDL, así como otras propiedades antioxidantes, anticarcinogénicas, antimutagénicas. De este modo, se concluye la necesidad de realizar más estudios con Morus nigra y principalmente más ensayos clínicos que evalúen la seguridad y eficacia en la utilización de esta planta.

Descriptores: Farmacología; Fitoterapia; Hiperlipidemias; Morus.

\section{INTRODUÇÃO}

Morus nigra $L$. é uma espécie vegetal pertencente ao gênero Morus, família Moraceae, que possui pelo menos 24 espécies e uma subespécie, com aproximadamente 100 variedades. Em algumas regiões do Brasil é conhecida como "amora-preta", entretanto, na região do Vale do São Francisco é popularmente denominada de "amora-miúra"

Morus nigra L. possui como origem a região do extremo oriente, é bastante utilizada na medicina tradicional chinesa. Foi trazida para o Brasil pelos imigrantes Japoneses, se adapta bem às condições de clima e solo. No vale do São Francisco, as folhas são utilizadas pela população na forma de chá (decocto), para o tratamento de diabetes, colesterol, problemas cardiovasculares, obesidade e gota ${ }^{2}$.

Vale ressaltar que, embora bastante utilizada na medicina popular, existem poucos estudos químicos e farmacológicos desta espécie. Entretanto, diferentes grupos de compostos químicos já foram identificados no gênero, como por exemplo, alcaloides, cumarinas, flavonoides, triterpenos e esteroides.

As folhas possuem uma grande importância econômica, por serem utilizadas como alimento para 
o bicho da seda, na região da Turquia ${ }^{3}$. Sob o ponto de vista etnobotânico, as folhas tem mostrado possuir ação diurética, hipoglicemiante, atividades hipotensoras e têm sido usadas para o tratamento do climatério, assim o último justificado pela quantidade de diferentes flavonoides, que possuem efeito fitoestrogênico.

A casca da raiz de amoreiras também tem sido muito utilizada como anti-inflamatório, antitússigeno e antipiréticos ${ }^{2,4}$. $\mathrm{O}$ extrato da raiz possui oxiresveratrol que, como outros fitoconstituintes, pode contribuir para a forte atividade inibitória tirosinase ${ }^{5}$.

O fruto da Morus nigra $L$ é utilizado para o tratamento de doenças hepáticas e renais. Além disso, podem ser consumidos como uma fruta fresca ou sob a forma de produtos de confeitaria, tais como compotas, geleias, sobremesas, suco, pasta e sorvetes $^{6,7}$.

Este estudo teve como objetivo realizar uma revisão bibliográfica das principais características botânicas, fitoquímicas e farmacológicas da Morus nigra.

\section{MATERIAL E MÉTODO}

Trata-se de revisão sistematizada da literatura, pois se constitui da análise da literatura publicada sobre as propriedades medicinais de Morus nigra $^{8}$. Para a busca foram utilizadas as bases de dados: MEDLINE - Medical Literature Analysis and Retrieval Sistem Online, SciELO - Scientific Eletronic Library Online e ainda o Portal de periódicos Capes e a Biblioteca Virtual em Saúde.

Foram utilizadas as palavras-chave em português e inglês: Morus nigra, farmacologia, fitoterapia, hipolipêmica. Foram incluídas no estudo as publicações realizadas entre 2003 e 2017 nos idiomas português e inglês nos formatos de artigo, dissertação e tese. Foram excluídos os artigos que não apresentavam o conteúdo disponível na íntegra para consulta, pois não estavam dentro do período estabelecido para a pesquisa e se tratassem de outras espécies de Morus sp. exceto a Morus nigra.

A partir das buscas foram avaliados os resumos das publicações e, as que se adequaram aos critérios do estudo, foram analisadas e seu conteúdo foi descrito e discutido para elaboração do presente estudo.

\section{RESULTADOS E DISCUSSÃO}

\section{Características botânicas}

A Morus nigra é uma planta popularmente conhecida por "Amoreira preta", de fácil adaptação e predominância nas regiões tropicais". Produz seus frutos com abundância na Ásia e está inteiramente aclimada e cultivada nas regiões sul e sudeste do Brasil. As plantas do gênero Morus têm importância e interesse de pesquisadores devido ao seu potencial fitoterápico e uso na medicina popular ${ }^{10}$.

A Morus nigra é uma espécie de árvore de médio porte que varia entre 4 a 5 metros de altura, possui cascas rugosas, escuras e copa grande ${ }^{11}$. Suas folhas medem cerca de 16 por $8 \mathrm{~cm}$, com margem serrilhada, base arredondada e ápice agudo ${ }^{7}$, possuem cor esverdeadas e pouco ásperas, e são ovaladas. As flores da Morus nigra possuem tamanho pequeno com coloração branco-amarelada, seus frutos são de coloração escura e, quando maduras são de coloração negra com a polpa vermelho-escura e aparência comestível ${ }^{11}$, possuem sabor agridoce característico e conhecido popularmente ${ }^{12}$.

\section{Composição química e propriedades farmacológicas}

Em vários estudos relacionados ao gênero Morus sp. foram relatados um amplo espectro de compostos químicos fenólicos, sendo incluídos estilbenos, flavonoides isoprenilados, cumarinas, cromonas e xantonas, além de outros compostos de Diels- Alder ${ }^{2}$. Grandes números de estudos têm sido realizados acerca dos compostos fenólicos encontrados em várias espécies do gênero Morus $s p$. em consequência de muitas atividades farmacológicas relacionadas a este composto ${ }^{13}$.

A Morus nigra tem sido relacionada a inúmeras atividades farmacológicas, de acordo com estudos realizados com extratos de seu fruto, folhas e raízes, dentre as atividades biológicas são citadas propriedades antioxidantes, anticarcinogênica e antimutagênica com grande capacidade na modificação da expressão gênica ${ }^{7}$.

As ações dos antioxidantes naturais encontrados nos frutos da Morus nigra $L$ ajudam na proteção do organismo contra radicais livres, diminuem a oxidação de lipídeos e consequentemente o progresso de algumas doenças crônicas, atuam inteiramente na neutralização da ação das classes reativas ou com sua função contribuir de forma indireta de sistemas enzimáticos. Estudos corroboram que o extrato livre de açúcar apresenta altos teores de flavonoides, compostos fenólicos, rutinas e ácido clorogênicos quando confrontados com a composição química da Morus alba ${ }^{11}$.

Lipídeos, ácidos graxos, vitamina C, minerais, são relatados ainda como presentes nos frutos das espécies do gênero Morus sp. pertencentes ao metabolismo primário das plantas ainda especificamente na Morus nigra antocianinas e alguns ácidos orgânicos ${ }^{4}$.

\section{Propriedades fitoquímicas e farmacológicas gerais da Morus nigra}

A Morus nigra é citada na medicina tradicional no tratamento de aftas, diarreia, dor de garganta, inflamações da boca, diabetes, problemas de menstruação e ovário. ${ }^{14}$ Ainda em estudo realizado com populares, foram citadas ações farmacológicas quando utilizaram a folha da planta na menopausa, reposição hormonal e "calorões"15. 
Os frutos de Morus nigra são utilizados em processos inflamatórios, para estancar hemorragias, como diurético e expectorante. As folhas são usadas como antídoto para envenenamento decorrente de picadas de animais peçonhentos; a casca do tronco é eficaz nas odontalgias. Estudos evidenciaram também que extratos da fruta de Morus nigra apresentam efeito protetor contra danos peroxidativos a biomembranas e a biomoléculas in vitro ${ }^{16}$.

Foi observado ainda que Morus nigra possui atividade anticancerígena contra a linhagem celular de câncer cervical humano (HeLa) in vitro. Estudo recente, evidenciou atividade de neuroproteção do efeito antidepressivo em camundongos, efeito atribuído principalmente ao ácido siríngico, principal constituinte fenólico presente no extrato aquoso de Morus nigra ${ }^{17,18}$.

\section{Atividade hipolipêmica da Morus nigra $L$.}

A dislipidemia é uma doença caracterizada por elevadas concentrações de lipídeos ou lipoproteínas na corrente sanguínea, determinada por fatores genéticos e ambientais. Este quadro clínico vem ao longo dos anos sendo relacionado a uma maior incidência de hipertensão e aterosclerose doença inflamatória crônica decorrente da deposição de placas lipídicas na parede arterial, que podem causar obstrução dos vasos sanguíneos ${ }^{19}$.

$$
\mathrm{O} \text { tratamento farmacológico das }
$$

dislipidemias constitui-se principalmente na utilização das estatinas, fibratos e resinas, entretanto, a aquisição de alguns destes fármacos é dificultada devido ao valor elevado e os de mais fácil acesso, como a amplamente utilizada Sinvastatina, possui diversas contraindicações tais como a doença hepática ativa, doença renal crônica em vigência de hemodiálise, uso concomitante a inibidores da CYP3A4 além de sua teratogenicidade que impossibilita o tratamento durante a gestação. Esta estatina pode ainda causar efeitos adversos de ordem gastrointestinal, neurológica, respiratória, hepática e músculoesquelética ${ }^{20}$.

As estatinas são inibidores competitivos da enzima HMGR bastante potentes, com valores de constante de inibição (Ki) na faixa nanomolar $(0,1$ a 2,3). Por exemplo, a afinidade de HMG-CoA redutase por mevastatina e lovastatina é, respectivamente, 7140 e 16700 vezes superior que pelo substrato, confirmando a presença de duas regiões de ligações fundamentais no sítio ativo da enzima, uma para ligação do grupo hidroximetilglutaril, no qual o substrato ou a porção de ácido 3,5-di-hidroxivalérico de estatinas se liga, e uma região adjacente hidrofóbica para complexação do grupo volumoso das estatinas ${ }^{21}$.

Desse modo, é de suma importância a realização de estudos que ampliem a possibilidade na utilização de novas terapias farmacológicas, sendo a fitoterapia, uma alternativa de fácil acesso e baixo custo. Diversos estudos relatam a atividade hipolipemiante da Amora negra. Pesquisa conduzida por Silva Júnior et al. ${ }^{22}$ demonstrou que o tratamento a curto prazo com o extrato etanólico das folhas de Morus nigra, reduz a hiperglicemia, melhora a tolerância oral à glicose, minimiza a dislipidemia e o estresse oxidativo e promove a redução do índice aterogênico em ratos diabéticos.

Zeni et al. ${ }^{23}$ testaram diversos tipos de extrato das folhas de Morus nigra em ratos e observaram efeito hipolipemiante com diminuição do índice aterogênico e do fator de risco cardíaco. Os autores associaram a ação hipolipêmica da Morus nigra ao ácido clorogênico e quercitina encontrados no extrato de suas folhas. Estes compostos podem atuar como adjuvantes no processo de queda dos níveis de colesterol total, triglicerídeos e LDL ${ }^{23}$.

Estudos realizados por $\mathrm{Li}$ et al. ${ }^{24}$ apontaram a modulação do metabolismo lipídico por ácido clorogênico na diminuição do colesterol total, triglicerídeos e LDL. Porém, os autores não observaram aumento dos níveis de HDL através da regulação da expressão do receptor ativado por proliferador de peroxissoma hepático (PPAR- $\alpha$ ).

Segundo Tavares ${ }^{25}$, receptores ativados por proliferadores de peroxissoma (PPARs) são fatores de transcrição pertencentes à família de receptores nucleares que regulam a homeostase da glicose, metabolismo de lipídeos e inflamação. Três proteínas, codificadas por genes distintos, têm sido identificadas: PPAR $\alpha$, PPAR $\beta$ e PPAR $\gamma$, as quais controlam a expressão gênica pela ligação a elementos responsivos específicos (PPREs) localizados na região promotora.

A atividade hipolipêmica de Morus nigra também foi avaliada em associação a Anredera cordifolia em ratas com hiperlipidemia. Os resultados deste estudo demonstraram que a combinação dos extratos atuam sinergicamente na diminuição dos níveis de colesterol total, LDL e triglicerídeos, contribuindo para elevação do HDL ${ }^{26}$.

Ensaio clínico randomizado realizado por Agahababaee et al. $^{27}$ avaliou 72 pacientes com dislipidemia, onde um grupo ingeria diariamente 300 $\mathrm{mL}$ de suco de amora negra (Morus nigra) com polpa e o outro grupo de pacientes ingeria uma dieta usual. Antes e após a intervenção foram mensuradas as concentrações de Apo AI e Apo B, colesterol total, HDL e triglicerídeos. Ao final do estudo foi verificado um aumento significante na Apo AI e no HDL, além de redução da pressão sistólica sanguínea no grupo que sofreu intervenção, demonstrando benefício na incorporação da amora negra na dieta de pacientes dislipidêmicos.

CONCLUSÃO

Com base na revisão realizada pode-se concluir que o consumo de frutos e chás extraídos das 
folhas da Morus nigra pela medicina tradicional tem obtido resultados satisfatórios relacionados às atividades farmacológicas/terapêuticas de doenças provenientes de estresse oxidativo, entre outras enfermidades. Fica nítido seu potencial antioxidante e hipolipemiante de acordo com os dados levantados, comprovado pela diminuição do estresse oxidativo, além da diminuição dos níveis de colesterol, LDL e triglicerídeos. Isto reforça a importância não só de aprofundar pesquisas da Morus nigra, como também de realizar ensaios clínicos desta e de outras espécies vegetais para a medicina tradicional no tratamento de doenças e complicações consequentes de valores elevados de colesterol, LDL e triglicerídeos.

\section{REFERÊNCIAS}

1. Almeida JRGS, Guimarães AL, Oliveira AP, Araújo ECC, Silva FS, Neves LF et al. Evaluation of hypoglycemic potential and pre-clinical toxicology of Morus nigra L. (Moraceae). Lat Am J Pharm. 2011; 30:96-100.

2. Oliveira ACB, Oliveira AP, Guimarães AL, Oliveira RA, Silva FS, Reis SAGB et al. Avaliação toxicológica pré-clínica do chá das folhas de Morus nigra L. (Moraceae). Rev Bras Pl Med. 2013; 15(2):244-49.

3. Aramwit P, Bang N, Srichana T. The properties and stability of anthocyanins in mulberry fruits. Food Res Int. 2010; 43(4):1093-97.

4. Pawlowska AM, Oleszek W, Braca A. Qualiquantitative analyses of Flavonoids of Morus nigra L. and Morus alba L. (Moraceae) fruits. J Agric Food Chem. 2008; 56(9):3377-80.

5. Zheng ZP, Cheng KW, Zhu Q, Wang XC, Lin ZX, Wang M. Tyrosinase inhibitory constituents from the roots of Morus nigra: a structure-activity relationship study. J Agric Food Chem. 2010; 58(9):5368-73.

6. Özgen M, Serçe S, Kaya C. Phytochemical and antioxidant properties of anthocyanin-rich Morus nigra and Morus rubra fruits. Sci Hort. 2009; 119(3):275-79.

7. Padilha MM, Moreira LQ, Morais FF, Araújo TH, Silva GA. Estudo farmacobotânico das folhas de amoreira-preta, Morus nigra L., Moraceae. Rev Bras Farmacogn. 2010; 20(4):621-26.

8. Li S, Orhan E. Chemical composition of white (Morus alba), red (Morus rubra) and black (Morus nigra) mulberry fruits. Food Chem. 2007; 103(4):1380-84.

9. Silva RAH. Estudo da ação do extrato bruto de Morus nigra L. (Moreaceae) e frações fenólicas sobre a atividade antimicrobiana e geração de espécies reativas do oxigênio e nitrogênio: in vitro com ensaios químicos, enzumáticos e celular [tese]. Araraquara: Universidade Estadual Paulista (UNESP), Faculdade de Ciências Farmacêuticas; 2012.
10.Tallini LR, Pedrazza GPR, Bordignon SAL, Costa ACO, Steppe M, Fuentefria A et al. Analysis of flavonoids in Rubus erythrocladusand Morus nigra leaves extracts by liquid chromatography and capillary electrophoresis. Rev Bras Farmacogn. 2015; 25(3):219-27.

11. Araujo CM. Análise da eficácia da polpa do fruto e do extrato das folhas de amoreira (Morus nigra 1.) sobre a modulação de marcadores metabólicos e marcadores do estado redox celular em um modelo experimental de diabetes tipo 1[tese]. Ouro Preto: Instituto de Ciências Exatas e Biológicas, Universidade Federal de Ouro Preto (UFOP); 2015.

12. Guizzo PL, Bredda TCC, Scarpa MVC, Navarro FF. Controle de qualidade e triagem fitoquímica da droga vegetal das folhas de Morus nigra L. (MORACEAE). Rev ciênc farm básica apl. 36(2):259-65.

13.Pereira CBP, Marin A, Dalmora SL, Necchi RMN, Moresco RN, Manfron MP. Atividade antiinflamatória e avaliação da toxicidade do extrato hidroetanólico de Morus alba (Moraceae). Rev ciênc farm básica apl. 2013; 34(1):43-6.

14. Boscolo OH, Valle LS. Plantas de uso medicinal em Quissamã, Rio de Janeiro, Brasil. Iheringia. 2008; 63(2):263-77.

15. Vendruscolo GS, Mentz LA. Levantamento etnobotânico das plantas utilizadas como medicinais por moradores do bairro Ponta Grossa. Porto Alegre, Rio Grande do Sul, Brasil. Iheringia. 2006; 61(1/2):83-103.

16. Queiroz GTD. Avaliação da atividade estrogênica do extrato hidroalcoolico de Morus nigra L. em ratas wistar (Rattus novergicus Berkenhout, 1769)[dissertação]. Juiz de Fora: Universidade Federal de Juiz de Fora (UFJF); 2011.

17.Qadir MI, Ali M, Ibrahim Z. Anticancer activity of Morus nigra leaves extract. Bangladesh $\mathrm{J}$ Pharmacol. 2014; 9(4):496-97.

18.Dalmagro AP, Camargo A, Zeni ALB. Morus nigra and its major phenolic, syringic acid, have antidepressant-like and neuroprotective effects in mice. Metab brain dis. 2017; 32(6):1963-73.

19. Franca E, Alves JGB. Dislipidemia entre crianças e adolescentes de Pernambuco. Arq Bras Cardiol. 2006; 87(6):722-27.

20.Xavier HT, Izar MC, Faria Neto JR, Assad MH, Rocha VZ, Sposito AC et Al. V Diretriz Brasileira de Dislipidemias e Prevenção da Aterosclerose. Arq Bras Cardiol. 2013; 101(4 supl.1):1-22.

21.Leitersdorf E, Safadi R, Meiner V, Reshef A, Björkhem I, Friendlander $\mathrm{Y}$ et al. Cerebrotendinous xanthomatosis in the Israeli Druze: molecular genetics and phenotypic characteristics. Am J Hum Genet. 1994; 55(5):907-15. 
22.Silva Júnior II, Barbosa HM, Carvalho DCR, Barros RA, Albuquerque FP, Silva DHA et al. Brazilian Morus nigra attenuated hyperglycemia, dyslipidemia, and prooxidant status in alloxaninduced diabetic rats. The Scientific World Journal. 2017, Article ID 5275813.

23.Zeni ALB, Moreira TD, Dalmagro AP, Camargo A, Bini LA, Simionatto EL et al. Evaluation of phenolic compounds and lipid-lowering effect of Morus nigra leaves extract. An Acad Bras Cienc. 2017; 89(4):2805-15.

24.Li B, Meng L, Lozano YF, Gaydou EM. Antioxidant activities of polyphenols extracted from Perilla frutescens varieties. Molecules. 2009; 14(1):133-40.

25.Tavares V, Hirata MH, Hirata RDC. Peroxisome proliferator-activated receptor gamma (PPARg): molecular study in glucose homeostasis, lipid metabolism and therapeutic approach. Arq Bras Endocrinol Metab. 2007; 51(4):526-33.

26. Sukandar EY, Safitri D, Aini NN. The study of ethanolic extract of binahong leaves (Anredera cordifolia [Ten.] Steenis) and mulberry leaves (Morus nigra L.) in combination on hyperlipidemic-induced rats. Asian J Pharm Clin Res. 2016; 9(6):288-98.

27. Agahababaee SK, Vafa M, Shidfar F, Tahavorgar A, Gohari M, Kteb D et al. Effects of blackberry (Morus nigra L.) consumption on serum concentration of lipoproteins, apo A-I, apo B, and high-sensitivity-C-reactive protein and blood pressure in dyslipidemic patients. J Res Med Sci. 2015; 20(7):684-91.
CONFLITO DE INTERESSES

Os autores declaram não haver conflitos de interesse.

AUTOR PARA CORRESPONDENCIA

Abrahão Alves de Oliveira Filho

abrahao.farm@gmail.com

Submetido em 03/04/2018

Aceito em 27/06/2018 